\title{
EL TIEMPO Y SU ANALOGÍA \\ CON LA NOCIÓN DE ESPACIO \\ EN LA FILOSOFÍA DE G. W. LEIBNIZ
}

\author{
LEONARDO RUIZ-GÓMEZ \\ Universidad Panamericana. Campus México
}

\begin{abstract}
RESUMEN. Leibniz, como muchos de sus contemporáneos, establece algunas conexiones conceptuales entre la noción de espacio y la noción de tiempo. Al ser el concepto de tiempo mucho menos explorado por Leibniz que el concepto del espacio, esta analogía nos puede abrir algunos caminos de interpretación valiosos. Sin embargo, es importante hacer un análisis detallado sobre los límites de la analogía y las notas características que se pueden extrapolar entre estos dos términos, pues no existe entre ellos un paralelismo estricto. El objetivo del presente artículo es señalar algunas notas esenciales del concepto leibniziano de tiempo a partir de su teoría del espacio. Para ello, se definirán las extrapolaciones válidas e inválidas entre estas dos nociones. Con ello se delineará una noción clara de tiempo, se mostrarán las posibles incongruencias que muestra este concepto respecto al sistema leibniziano y, finalmente, se ofrecerán algunas posibles vías de solución a estas inconsistencias.
\end{abstract}

PALABRAS CLAVE: Leibniz, tiempo, espacio, analogía, monadología.

\section{Leibniz's notion of time and its analogy to his notion of space}

ABSTRACT. Leibniz, as many of his contemporaries, establishes some conceptual connections between the notion of space and the notion of time. Since the concept of time is much less explored by Leibniz than the concept of space, this analogy could offer some clues to understand the first. However, it is important to analyse the limits of this analogy and the properties that can be inferred between the two notions, since there is no strict parallelism among them. The aim of this paper is to identify some essential elements of the Leibnizian concept of time in connection with his theory of space. First, I shall determine which extrapolations are valid between these two terms. Then, I shall provide a clear notion of time, as well as some possible contradictions within the Leibnizian system in this regard. Finally, some possible ways of solutions to these inconsistencies will be offered.

KEY WORDS: Leibniz, time, space, analogy, monadology.

La polémica entre Leibniz y Newton es considerada el locus clásico de la discusión entre las tesis relativistas y absolutistas del espacio. Esta disputa, comandada por el amigo de Newton, Samuel Clarke, estableció los términos en los que se discutió la naturaleza del espacio durante los siguientes dos siglos. Leibniz se convertiría así en uno de los defensores más sofisticados del relativismo del espacio en la época moderna.

El tema del tiempo parece no poseer, en cambio, las mismas credenciales dentro del pensamiento del filósofo alemán. En efecto, mientras que Leibniz abordó de manera prolija el tema del espacio — si bien ya en una etapa tardía de su vida-, el concepto del tiempo aparece con mucha mayor discreción dentro de la obra leibniziana. Es evidente, sin embargo, que dicho concepto comenzó a adquirir fuerza en los últimos años de la vida del sabio alemán y que fue precisamente éste el siguiente paraje del siempre disperso y omniabarcante pensamiento leibniziano. 
Esta última afirmación, de tintes más bien cabalísticos que académicos, no es del todo disparatada cuando se atiende a la laguna sistemática que la cuestión del tiempo representa dentro del constructo teórico leibniziano. Cuando digo que se trata de una laguna sistemática no sólo quiero dar a entender que es un asunto que no abordó suficientemente el filósofo alemán dentro de su casi inabarcable obra, sino que es un tema que exige explicación a la luz del resto de ideas que sí desarrolló Leibniz.

El presente trabajo tiene, en consonancia con estas ideas, una consigna muy específica, por no decir modesta: establecer la relación entre lo que Leibniz dijo sobre el tiempo y las exigencias que el resto del sistema leibniziano parecen imponer a esta noción. Para ello, comenzaré mi exposición con un análisis somero de las referencias textuales que se tienen sobre el tiempo en la obra leibniziana (§1). Después, haré un breve bosquejo de la noción leibniziana de espacio que, como se verá, está íntimamente ligada a la de tiempo (§2). Posteriormente analizaré la analogía que establece Leibniz entre estas dos nociones con el fin de extraer algunas conclusiones sobre la naturaleza del tiempo (§3). Será momento después de señalar algunos límites que pueden hacer problemática la comprensión del paralelismo entre el espacio y el tiempo (§4), además de reseñar algunas soluciones que otros comentaristas han ensayado para delimitar estos conceptos (§5). Finalmente, intentaré dar algunas posibles soluciones propias para resolver las aporías presentadas a lo largo de la exposición (§6).

\section{El TIEMPO COMO «ORDEN DE LAS EXISTENCIAS SUCESIVAS»}

Cuando digo que Leibniz es parco en sus explicaciones en torno al tiempo no es por falta de menciones del término en el corpus, sino por la casi exasperante repetitividad de sus alusiones al tema en las que, paradójicamente, resulta ser profundamente inconsistente en su terminología.

Leibniz oscila entre distintas variaciones: el tiempo es definido a veces como un "orden de existencia no simultánea o sucesiva» ${ }^{1}$, como el «orden de las sucesiones» $»^{2}$, como un «orden general de las mutaciones y de la existencia de los incompatibles» ${ }^{3}$, el «orden de los fenómenos sucesivos ${ }^{4} »$, «orden de

1 Leibniz a Remond, s/f, G III, p. 612; Conséquences métaphysiques du principe de raison, C p. 14; Leibniz a Bernoulli, 7 de junio de 1716, GM III, p. 964, 1 de julio de 1704, GM III, p. 756; Leibniz a Clarke, 2 de junio de 1716, G VII, p. 378; Leibniz a Des Bosses, 31 de julio de 1709, G II, 378-379; De tempore locoque, duratione ac spatio, AVI, 4, p. 1641; Nouveaux Essais..., II, cap. 4, §5, G V, p. 115.

2 Leibniz a Clarke, 25 de febrero de 1716, G VII, p. 363; Leibniz a Des Bosses, 5 de febrero de 1712, G II, p. 438.

3 Leibniz a Bernoulli, 9 de abril de 1715, GM III, p. 939.

4 Leibniz a Des Bosses, 16 de junio de 1712, G II, pp. 450-451. 
las posibilidades no coexistentes» $»^{5}$ "orden continuo de coexistencia» ${ }^{6}$. El leitmotiv es claro: el tiempo es un cierto tipo de orden cuya nota característica es la sucesión. Pero ese orden encuentra por referencia elementos demasiado variados como para no levantar sospechas. A veces, se trata del orden de «las cosas»; a veces, de «las posibilidades»; a veces, «de los fenómenos»; a veces, Leibniz dice sin más «los incompatibles». En ciertas ocasiones, se resalta el carácter «fenoménico» del tiempo; en otras, el carácter «ideal» y «continuo». Cuando se observa, por ejemplo, que «ideal»y «fenoménico» pueden tratarse de registros completamente opuestos en el pensamiento leibniziano, o que las «posibilidades» rebasan en extensión lógica, como es obvio, el ámbito de los «fenómenos actuales» o de las «cosas», se comprende que Leibniz nos está regalando un rompecabezas de difícil solución.

Es reconfortante, al menos, que en la mayoría de los textos en los que Leibniz menciona al tiempo, se habla de éste en franco paralelismo con el espacio; en efecto, Leibniz pocas veces habla del tiempo sin hacer referencia a su concepto espejo: el «orden de coexistencia», o espacio. Tentativamente podríamos encontrar un cierto alivio en esta reiterada analogía: descifrando el espacio, será posible esclarecer los misterios que el concepto de tiempo guarda en las expresiones leibnizianas. El contento es breve, sin embargo, cuando se descubre que las mismas inconsistencias que se encuentran en la formulación del tiempo se encuentran en la formulación del espacio ${ }^{7}$. Ahí también Leibniz introduce por igual a los cuerpos que a los fenómenos, a las posibilidades que a las cosas; todas ellas, esta vez, coexistentes, y no sucesivas. Por fortuna, y a diferencia del tiempo, Leibniz sí aporta un tratamiento más o menos detallado del espacio que permite limar varias aristas en esta materia. Este tratamiento más prolijo permite comprender el sentido de las diversas expresiones - a primera vista inconsistentes- sobre la naturaleza del espacio en el marco de su pensamiento.

Bajo esta luz, sería fácil pensar que quien descifre el significado de la noción de espacio tiene la partida ganada en el territorio menos amigable del tiempo. Considerando provisionalmente esta afirmación como verdadera, pasaré a hacer un breve bosquejo de lo que, en mi opinión, es la teoría del espacio leibniziana ${ }^{8}$.

5 Leibniz a De Volder, 20 de junio de 1703, G II, p. 253; 30 de junio de 1704, G II, pp. 268-269; Response aux reflexions..., G IV, p. 568; Leibniz a Schulenburg, s/f, GM VII, p. 242.

6 Leibniz a De Volder, 31 de diciembre de 1700, G II p. 221; Nullum quidem librum..., G IV, p. 393-394.

7 Las ambigüedades sobre el espacio son equivalente en muchos sentidos a las del tiempo. Para un análisis detallado de las inconsistencias en el tratamiento del espacio, cfr. Ruiz-Gómez, Leonardo, El concepto leibniziano de espacio. La polémica con Clarke y el newtonianismo, EUNSA, Pamplona, 2014, p. 296, n. 3.

8 Por cuestiones de espacio, no podré defender aquí suficientemente la lectura que hago de los textos leibnizianos, sino que me limitaré a exponerla. Una extensa descripción de mi interpretación del concepto de espacio en la obra leibniziana puede consultarse en RuizGómez, El concepto leibniziano de espacio, c. IV. 


\section{LA TEORÍA LEIBNIZIANA DEL ESPACIO}

Como es bien sabido, la obsesión metafísica de Leibniz era la solución de lo que él llamaba el laberinto del continuo. Esto es: ¿cómo explicar la entidad, la subsistencia ontológica, de aquello que es infinitamente divisible?

El mundo leibniziano está constituido exclusivamente por mónadas, sus percepciones y el tránsito entre estas percepciones en la mónada9. Cualquier otra realidad que se presente en los fenómenos debe encontrar su explicación en estos elementos ${ }^{10}$. Así, el continuo, siendo infinitamente divisible, no puede ser existente en sentido propio; y, sin embargo, debe estar fundado en alguna entidad del ámbito transfenoménico.

En el lenguaje leibniziano, fenómenos y percepción son cara de la misma moneda: los fenómenos se constituyen en la percepción de la mónada. Leibniz ofrece en su Monadología una famosa definición de su noción de percepción: es el «estado transitorio que envuelve y representa una multitud en la unidad o en la sustancia simple» ${ }^{11}$. La percepción es, pues, una actividad sintética: representa la pluralidad del mundo en la simplicidad del yo. Pero el carácter sintético de la mónada determina a esta representación a ser confusa: no hay síntesis sin pérdida y a esta pérdida de información, Leibniz la llama "confusión»"

Sería útil en este punto comparar la percepción de la mónada con el conocimiento divino del mundo. Para Dios, no hay propiamente fenómenos, precisamente porque su conocimiento de las sustancias es directo y no sintético. Dios aborda lo infinito en acto. Las sustancias finitas en cambio, se dirigen al infinito confusamente ${ }^{13}$. Dios conoce - usando un término anacrónico (y paradójico) - nouménicamente; las sustancias finitas, en cambio, conocen fenoménicamente.

Así, el carácter finito de la percepción monádica tiene como efecto una representación confusa. Esta confusión no es otra cosa sino la percepción difusa de un conjunto de propiedades del resto de las mónadas que son en algo

9 «Considerando adecuadamente las cosas, debe decirse que nada hay en las cosas sino sustancias simples y en ellas percepciones y apetitos». Leibniz a De Volder, 30 de junio de 1704, G II, p. 270. Cfr. también Principes de la nature et de la grâce..., G VI, p. 598.

10 Cfr. Garber, Daniel, Leibniz: Body, substance, Monad, Oxford Univ. Press, Oxford, 2009, p. 317.

11 Monadologie, §14, G VI, p. 608. Cfr. también, Principes de la nature et de la grâce..., §2, G VI, p. 598; Esta definición de percepción se encuentra ya esbozada en textos de la época del Discours de Métaphysique (1686): cfr. Discours de Métaphysique, A VI, 4B, p. 1542.

12 Discours de Métaphysique, A VI, 4B, p. 1542; Monadologie, §60, G VI, p. 617.

13 Leibniz explica estas formas de «conocimiento» mediante una metáfora. Dios conoce el mundo «icnográficamente», las mónadas lo perciben «escenográficamente»: «Pues las escenografías son diferentes en función del sitio del espectador, mientras la iconografía, o representación geométrica, es única. Dios ve las cosas exactamente como son según la verdad geométrica, aunque él sabe también de qué manera aparece cada cosa a cada uno, y así contiene en sí eminentemente todas las otras apariencias». Leibniz a Des Bosses, 5 de febrero de 1712, G II, p. 438. 
homogéneas. Así es como se trata de salvar el laberinto del continuo: dada la estructura finita de la mónada percipiente (lo único que es verdaderamente uno), ciertas cualidades son representadas como continuas: por ejemplo, la difusión de la fuerza pasiva de las mónadas engendra el continuo material ${ }^{14}$, y la difusión de la fuerza activa da origen al movimiento continuo ${ }^{15}$.

Un tipo de difusión es particularmente importante para nuestros intereses. Leibniz afirma en algunos textos que la difusión del situs o punto de vista de las mónadas engendra la extensión continua ${ }^{16}$. La noción de punto de vista de la mónada es bien conocido: si las mónadas representan a la totalidad del mundo, lo hacen desde una perspectiva particular. Esto es, nuevamente, fruto de su finitud (de lo contrario, "cada mónada sería una divinidad» ${ }^{17}$ ). Ahora bien, esta representación se distingue por ciertos grados de distinción y, según afirma Leibniz, estos grados de distinción están íntimamente relacionados con una cierta distancia entre la mónada representada y la mónada que la percibe ${ }^{18}$.

Es bien sabido, sin embargo, que Leibniz sostiene una posición relativista del espacio, y que hablar de distancias absolutas y reales entre las mónadas sería contradictorio en su sistema. Esto quiere decir que la distancia de la que habla Leibniz debe entenderse en un sentido metafórico, ya que la representación monádica (lo real) no puede estar determinada por propiedades espaciales en sentido estricto (lo derivado). Se debe postular así una noción de «distancia representativa» que será el fundamento de la distancia fenoménica, esto es, de las propiedades espaciales.

Así, la extensión y el espacio están fundamentados, precisamente, en las distancias representativas entre las mónadas. En la difusión del punto de vista de las mónadas se engendra un continuo extensional en donde comparecerán las demás propiedades monádicas (fuerza activa y fuerza pasiva, principalmente). El esquema final seguiría grosso modo el siguiente modelo. Las mónadas y sus percepciones son lo único que existe realmente. Las mónadas se perciben entre sí desde un punto de vista (situs) y con un punto de vista. Cuando se percibe difusamente una pluralidad de mónadas, se presenta en los fenómenos la extensión como un continuo, esto es, la difusión del situs. Cuando se realiza una abstracción combinatoria de las posiciones posibles a partir de la extensión fenoménica, aparece el concepto de espacio, que es ideal, continuo y abstracto: es el orden posible de coexistencia.

Resumiendo aún más: las mónadas están fuera del espacio y no tienen relaciones espaciales entre sí, sino representativas; los fenómenos se representan extensionalmente, en un continuo; la abstracción de las posibles relaciones de situación en este continuo extensional engendra el concepto de espacio.

\footnotetext{
14 Cfr. Leibniz a Bernoulli, 30 de junio de 1712, GM III, p. 889.

15 Cfr. Specimen Dynamicum I, GM VI, pp. 237-238.

16 Cfr. Entretien de Philarete et d'Ariste..., G VI, p. 585; Specimen Dynamicum I, GM

17 Monadologie, $\$ 60, \mathrm{G}$ VI, p. 617.

18 Cfr. Monadologie, §60, G VI, p. 617.
} VI, p. 237. 


\section{LA ANALOGÍA ENTRE EL ESPACIO Y EL TIEMPO}

Este apresurado esquema nos permitiría, dada la analogía espacio-tiempo que se mencionó anteriormente, sacar conclusiones importantes respecto al tiempo y las relaciones temporales. La primera de ellas es que las mónadas deberán estar fuera del tiempo así como están fuera del espacio y, sin embargo, deberá haber cualidades en ellas que sean isomórficas con la temporalidad de los fenómenos. La segunda es que la temporalidad es fenoménica, esto es, que así como los fenómenos poseen extensión, se atribuye también a estos una duración. La duración debe ser, entonces, al igual que la extensión, una cierta difusión. Finalmente, la consideración abstracta de las posibles sucesiones deberá constituir el tiempo abstracto e ideal, del mismo modo en que la consideración abstracta de todas las relaciones de situación constituye el concepto de espacio absoluto.

Es oportuno preguntarnos ahora sobre los límites de esta analogía. El mismo Leibniz advierte en una carta a Samuel Clarke que «de la extensión a la duración non valet consequentia ${ }^{19}$. Esta advertencia debería hacernos dudar sobre la validez de la analogía a la que se recurre aquí. Bien se podría pensar también que se trata sólo de una argumentación retórica en el contexto de la pelea de Leibniz contra los newtonianos, lo que no sería extraño en lo absoluto. Me parece plausible, sin embargo, que, como en toda analogía, los analogados posean elementos en común y difieran en otros.

¿Cuáles son, pues, los elementos en los que difieren la extensión y la duración? Uno es evidente: en un caso, lo que se difunde es coexistente (en la extensión); en otro caso, lo que se difunde es sucesivo (en la duración). Sin embargo, el concepto de «sucesión» no puede hacer referencia a ninguna especie de temporalidad primigenia, pues implicaría una definición recursiva. ¿Qué podría significar que una pluralidad de elementos sea sucesiva al margen de cualquier criterio de temporalidad?

Tal vez sea un texto escrito en los últimos años de vida de Leibniz (17141716), el Initia rerum mathematicarum metaphysica, la fuente primaria que más haya sido utilizada por la literatura secundaria para elucidar el concepto de tiempo. Ahí, Leibniz se ocupa de establecer el fundamento de la sucesión temporal.

"Si se supone que existen muchos estados de cosas que no envuelven ninguna oposición, se dice que existen simultáneamente. Así, negamos que sea simultáneo lo que tuvo lugar el año pasado y lo que tuvo lugar este año, pues envuelven estados opuestos de la misma cosa.

Si de éstos [estados] que no son simultáneos uno envuelve una razón del otro, se tiene a aquél como primero, a éste como posterior. Mi estado anterior envuelve la razón de que mi estado posterior exista. Y como mi estado anterior — dada la conexión de todas las cosas- también envuelve el estado

19 Leibniz a Clarke, G VII, p. 408 (L.V.74). 
anterior de las otras cosas, así mi estado anterior también envuelve la razón del estado posterior de las otras cosas; y por tanto, es de hecho anterior a éste. Y así, cualquier cosa que existe es o bien anterior, o bien posterior, o bien simultánea a otros existentes» ${ }^{20}$.

Si Leibniz había basado la representación fenoménica del espacio en una distancia representativa, aquí define las relaciones temporales fenoménicas en términos de simultaneidad, anterioridad y posterioridad representativas. Utilizo el término "representativa» porque en sede monádica los «estados de cosas» de los que habla Leibniz sólo pueden comprenderse como percepciones determinadas del universo en cada mónada. Bajo estos términos, la simultaneidad estaría definida por la no-contradicción de los estados representativos; esto ya sería suficiente para entender el término "coexistencia» que forma parte de la definición de espacio ${ }^{21}$.

Sobre la posibilidad de establecer una serie no temporal como fundamento de una secuencia temporal, el mismo Leibniz parece establecerlo en su correspondencia con De Volder:

«Prosigue usted: «en una serie (como la de los números) nada se concibe sucesivo" ¿Y, con esto, qué? Yo no digo que una serie sea una sucesión, sino que una sucesión es una serie, y que tiene en común con todas las demás series el que la ley de la serie muestra hasta dónde se ha de llegar progresando en ella o, dicho de otro modo, puesto el inicio y la ley de progreso se producen los términos por orden, tanto que el orden y el inicio lo sean sólo por naturaleza como que lo sean también en el tiempo» ${ }^{22}$.

Una primera objeción surge a la vista: la experiencia cotidiana demuestra que no todos los estados no-contradictorios son simultáneos. ¿Qué impediría, por ejemplo, que el estado de cosas en el que esta habitación está llena sea sucesivo con el estado de cosas en el que la habitación contigua esté llena? El hecho «el salón A está lleno» no es contradictorio con el hecho «el salón B está lleno»y, sin embargo, tales estados no tienen que ser necesariamente simultáneos (como parecería pedir la definición leibniziana). El problema es de perspectiva. El objetor se equivoca — pensará Leibniz- en que realiza un análisis local completamente arbitrario; porque el salón A —estando llenono es del todo idéntico cuando el salón B está lleno y cuando el salón B está

20 Initia rerum mathematicarum metaphysica, GM VII, p. 18. Existen antecedentes mucho más tempranos de este tratamiento, si bien con importantes variantes: cfr. Definitiones notionum metaphysicarum atque logicarum, A VI, 4, pp. 628-629; Divisio terminorum ac enumeratio attributorum, A VI, 4, pp. 561-563.

21 Discrepo aquí de Michael Futch, quien afirma que lo simultáneo puede definirse como lo espacialmente conectado. Cfr. Futch, M., Leibniz's Metaphysics of Time and Space, Springer, Dordrecht, 2008, p. 124. Ciertamente lo «espacialmente conectado»y lo «simultaneo» son términos coextensivos, pero la noción de simultaneidad es anterior a la de espacio (pues entra en la definición de éste) y, en esa medida, no puede definirse la simultaneidad en términos espaciales sin caer en recursividad.

22 Leibniz a De Volder, 21 de enero de 1704, G II, p. 263. 
vacío. Debe haber diferencias —infinitesimales, si se quiere- que determinen al salón A cuando B está lleno de modo tal, que se vuelva irreconciliable y por tanto sucesivo, con el estado de A cuando B está vacío. La conexión de todas las $\operatorname{cosas}^{23}$ que postula Leibniz tiene como consecuencia que se defina con precisión un «ahora universal $»^{24}$. Si ampliamos la perspectiva lo suficiente, se observa que no hay nunca un estado de cosas que se repita; no en la medida en que cada evento local envuelve el infinito. En segunda instancia, se define la anterioridad y la posterioridad en términos no temporales. La noción clave aquí es la de «razón». Hay que decir que Leibniz define los hechos contingentes como aquéllos cuya verdad implica un análisis infinito de sus razones (o bien, que tiene razones infinitas) ${ }^{25}$. De esa forma, si $x$ se cuenta dentro del análisis infinito de las razones de $y, x$ se presenta en los fenómenos como anterior a $y^{26}$.

Así, se comprende un «ahora universal» o una «línea de simultaneidad» que queda definida por todos los estados posibles de cosas que no envuelven contradicción ${ }^{27}$. El resto de los estados que sí implican contradicción queda estratificado en dos bloques. Uno - el del pasado-cuyos elementos se cuentan entre las razones de los estados que se encuentran en la línea de simultaneidad;

\section{Cfr. Monadologie, §61, G VI, p. 617}

24 Hay aquí una indicación que debería prevenir a los entusiastas de equiparar las tesis leibnizianas con la relatividad moderna. En efecto, la relatividad einsteiniana descarta la existencia de un «ahora» extendido a lo largo del espacio, que la relatividad leibniziana asume sin cortapisas. En efecto, se podría pensar en la teoría leibniziana como en una relatividad segmentada, en donde el espacio y el tiempo abrazan órdenes distintos de relación. La relatividad de Einstein supone, en cambio, una relatividad en bloque de relaciones espaciotemporales.

25 «[E]n las proposiciones contingentes, el análisis se vuelve infinito, y procede de razón en razón de tal manera que nunca se alcanza una completa demostración; esto no significa sin embargo que no haya en la base una razón de verdad, aunque sólo sea comprendida perfectamente por Dios, dado que es el único que con una sola mirada de su mente penetra la serie infinita de razones». De contingentia, A VI, 4B, p. 1650.

${ }_{26}$ Leibniz considera la objeción inversa a la aquí propuesta: dos cosas se pueden percibir simultáneamente, y no ser, sin embargo, contradictorias: «Pero no sólo conocemos aquellos entes que se perciben simultáneamente, sino también aquellos que percibimos en sucesión, con tal de que se postule que durante el tránsito de la percepción del uno a la percepción del otro el primero no perece, ni recae el segundo. De aquella hipótesis se sigue que ambos coexisten al tiempo en que alcanzamos el segundo; de ésta, que ambos existían ya cuando considerábamos el primero». Cfr. Initia rerum mathematica..., GM VII, p. 25. (tr. Ezequiel de Olazo)

27 Rescher define la simultaneidad como la semejanza entre estados monádicos. Si una mónada A posee un estado $\mathrm{s}_{1}$ cuya semejanza es máxima con la del estado $\mathrm{s}_{2}$ de la mónada B, entonces A y B son simultáneas en $\mathrm{s}_{1} \mathrm{y} \mathrm{s}_{2}$. Cfr. Rescher, Nicolas, The Philosophy of Leibniz, Prentice Hall, New Jersey, 1967, p. 85. No encuentro, sin embargo, motivos para aprobar esta definición que, por una parte, carece de evidencia textual y, por otra parte, abona poco a la comprensión del concepto de simultaneidad en la medida en que es ambigua la noción de semejanza en este contexto y que, como se verá, presupone un «tiempo público» donde se ubican los estados de las mónadas. Contra la postura de Rescher en este punto, cfr. también Mates, Benson, The Philosophy of Leibniz, Oxford Univ. Press, New York, 1986, p. 230. 
otro, el del futuro, cuyos elementos cuentan entre sus razones a los estados de la línea de simultaneidad ${ }^{28}$.

Quisiera señalar un hueco en el análisis que he presentado aquí y que es, me parece, heredado de una omisión de Leibniz en esta materia. Hemos visto cómo el continuum temporal puede ser definido por relaciones de causalidad y de contrariedad lógica. Se decía que el conjunto de estados contradictorios podía distribuirse asimétricamente entre aquéllos que formaban parte del pasado y aquéllos que formaban parte del futuro. Con esta caracterización se puede dar cuenta del desenvolvimiento temporal de cada mónada en términos de sucesión y coexistencia de estados. Este modelo no parece dar razón, sin embargo, del fenómeno del «ahora» (no entendido, desde luego, como límite del tiempo, al modo aristotélico, sino como "presente fenoménico»). No es lo mismo dar razón de la coexistencia en términos lógicos mediante «líneas de simultaneidad», que explicar la percepción subjetiva de encontrarse en una precisa línea de simultaneidad y no en otra. Esto es, si la filosofía leibniziana trata de poner en la mónada aquello que exigen los fenómenos como requisito, ¿qué habría en la mónada que explique la sensación subjetiva del presente o ahora fenoménico? Como dije, el modelo que he propuesto aquí no da respuesta a esta pregunta y, hasta donde he sido capaz de investigar, tampoco Leibniz ofrece solución alguna ${ }^{29}$.

Aquí hay una segunda diferencia entre el concepto de tiempo y espacio: el espacio es absolutamente simétrico, de manera que una «distancia representativa» no posee un sentido definido. En el Universo leibniziano no hay arriba ni abajo, derecha o izquierda. Sí hay, en cambio, una direccionalidad en el tiempo. El pasado y el futuro tienen un significado real. Podría objetársele a Leibniz que esta discrepancia entre el concepto de tiempo y espacio es, cuando menos, sospechosa; debe considerarse, sin embargo, que esta diferencia es dada en los fenómenos y Leibniz sólo está buscando hacer una fundamentación monádica de éstos.

28 Michael Futch advierte adecuadamente (contra Cover y Arthur) que el modelo leibniziano del tiempo debe entenderse en dos distintos momentos: primero se establece lo sucesivo como aquello que envuelve contradicción y después se ordenan estos estados sucesivos en anterioridad y posterioridad. A lo que se opone Futch es a que se defina directamente lo temporalmente anterior como aquello que envuelve una razón o causa, pues esto implicaría que dos eventos simultáneos no podrían tener una relación causal, como de hecho sucede. Así, lo anterior temporalmente debe entenderse como «el estado no-simultaneo que implica una razón de otro estado». Cfr. Futch, Michael, Leibniz Metaphysics of Time and Space, pp. 118-121.

${ }_{29}$ Este tema ha sido tratado en la bibliografía secundaria, y se reduce al debate entre las Teorías-A o Teorías-B del Tiempo. Las primeras asignan una significado ontológico real a los conceptos de pasado, presente y futuro; mientras que las segundas intentan describir el tiempo en términos de simple anterioridad y posterioridad. No me atrevería a afirmar que Leibniz se decanta por una Teoría B del tiempo, simplemente creo que carece de elementos para hacer la reconstrucción de una Teoría A. Sobre esto hay una amplia discusión en Di BELLA, Stefano, "Time, Contradiction and Change in Leibniz: Some Reflections», en: Li, Wenchao, et al (eds.), "Für unser Glück oder das Glück anderer» Vorträge des X. Internationalen LeibnizKongress, vol. IV, Olms, Hildescheim, 2016, pp. 263-267; Fuтch, M., Leibniz's Metaphysics of Time and Space, p. 142. 
Así las cosas, la analogía entre el espacio y el tiempo en el pensamiento leibniziano funcionaría de la siguiente manera: las mónadas no se encuentran ni en el espacio ni en el tiempo. Sus propiedades, sin embargo, se difunden en la percepción de otras mónadas generando un continuo espacio-temporal. La abstracción que se realiza de estas propiedades, considerando exclusivamente el orden en el que se presentan en los fenómenos, engendra el espacio y el tiempo ideal. Los analogados se diferencian, sin embargo, en que el tiempo expresa fenoménica o idealmente la difusión de elementos sucesivos; el espacio o extensión, de elementos coexistentes. Por otra parte, el tiempo y la duración son asimétricos, pues su fundamentación metafísica en la relación asimétrica entre causa y causado genera una verdadera flecha del tiempo, mientras que el espacio y la extensión son absolutamente simétricos ${ }^{30}$.

Estos dos reparos que encontramos en la analogía entre espacio y tiempo no son problemáticos, pues parecen estar contemplados dentro del sistema leibniziano. Leibniz no parece estar interesado en establecer una continuidad entre espacio y tiempo, sino en establecer un paralelismo aproximado de estas nociones. Hay, sin embargo, otros límites de la analogía que no parecen estar contemplados en los textos leibnizianos y que impiden que la noción de tiempo engarce dócilmente con el resto del sistema. Son estas dificultades las que nos ocuparán ahora en el resto del análisis.

\section{LOS LÍMITES DE LA ANALOGÍA Y LAS INCONSISTENCIAS DEL SISTEMA}

Quien lee la Monadología podría esperar encontrar algo de lo aquí dicho respecto a la relación entre las mónadas, el espacio y el tiempo. La verdad es que este texto canónico ayuda poco a la comprensión de estas nociones (y tampoco lo hacen documentos imprescindibles como el Nuevo Sistema o Los principios de la naturaleza y de la gracia). Sin embargo, es posible reconciliar lo dicho ahí sobre las mónadas con las líneas generales de la teoría del espacio leibniziana tal como ha sido esbozada en la segunda sección del presente trabajo. Es en la Monadología donde Leibniz formula con mayor patencia su tesis sobre el «punto de vista» monádico ${ }^{31}$, y es también ahí donde excluye, en

30 Anapolitanos identifica con razón otras dos diferencias, a parte de la direccionalidad del tiempo: la multidimensionalidad del espacio frente la unidimensionalidad del tiempo, y el carácter estático del espacio frente el carácter dinámico del tiempo. Otra discrepancia interesante: si fuera posible el vacío—si bien Leibniz no lo admite más que como hipótesisen el caso del espacio sería observable, pero un vacío en el tiempo sería imperceptible. Cfr. Anapolitanos, Dionysios, Leibniz: Representation, Continuity and the Spatiotemporal, Kluwer Academic, Dordrecht, 1999, pp. 138-140.

31 «Y así como una ciudad contemplada desde diferentes lados parece enteramente otra y se halla como multiplicada según la perspectiva, ocurre también que, debido a la multitud infinita de las sustancias simples, hay como otros tantos universos diferentes que, sin embargo, no son más que perspectivas de uno sólo según los diferentes puntos de vista». Monadologie, §57, G VI, p. 616. 
buena medida, a las mónadas del ámbito de la extensión ${ }^{32}$. No ocurre lo mismo con el tiempo, que, en cierto grado, brilla por su ausencia en la Monadología. No sólo eso: Leibniz introduce la noción de cambio interno de la mónada en varios de sus parágrafos; esto pone en un serio aprieto la idea de que la mónada se encuentra fuera del tiempo, la cual era una de las conclusiones directas de la analogía con el espacio. Se lee, en efecto, en la Monadología:

«De lo que acabamos de decir se sigue que los cambios naturales de las mónadas proceden de un principio interno, puesto que una causa externa no puede influir en su interior ${ }^{33}$.

Si el principio de cambio es un principio interno a la mónada, es difícil no concebir a la mónada como un devenir continuo de representaciones cuya condición de posibilidad es un tiempo interno anterior a las representaciones mismas. Pero una consideración de este tipo rompería de tajo con la analogía entre espacio y tiempo. En efecto, se tenía al espacio por un ente derivado (no real), precisamente porque no había en la naturaleza de la mónada ninguna relación propiamente espacial y, por lo tanto, la extensión o el espacio estaban en el nivel fenoménico e ideal. Pero si el tiempo está en el corazón mismo de la mónada, ¿cómo ha de suponerse a la mónada misma fuera del tiempo? Y si se considera, además, como hace Leibniz, que este cambio en las mónadas está armónicamente preestablecido, ¿no habría que asumir, como quería Newton, un «tiempo absoluto, verdadero y matemático» que «fluye uniformemente» $\mathrm{y}$ que es anterior a todo cambio y todo ser? ${ }^{34}$

Hay un problema adicional: es fácil explicar la naturaleza del espacio a partir de la difusión de las diferentes situaciones de las mónadas, en buena medida, porque cada "punto de vista» está fundamentado en un individuo real. No se cae en el laberinto del continuo porque se explica la pluralidad a partir de unidades reales (mientras que el laberinto del continuo se trata, precisamente, de tratar de encontrar las unidades a partir de un continuo). Sin embargo, en el caso del tiempo, lo que se difunde - esto es, lo estados sucesivos de las mónadas- no son individuos reales, sino estados diversos de un mismo individuo. El cambio monádico, entendido como pluralidad de estados, no está fundamentado en individuos reales; a menos que se esté dispuesto a definir a la mónada como un simple conglomerado de estados diversos.

32 «Ahora bien, donde no hay partes no hay extensión, figura ni divisibilidad posibles». Monadologie, §2, G VI, p. 607.

33 Monadologie, \$12, G VI, p. 608.

34 Principia I, definitiones VIII, escolio, p. 6[46]. Curiosamente, Richard Arthur considera este principio de cambio como la solución al problema de que el tiempo reciba una consideración abstracta y estática. Para Arthur, la descripción del tiempo en términos de estados contradictorios requiere de un motor que produzca el cambio de un estado a otro. Cfr. Arthur, Richard, "Leibniz's Theory of Time», en: Okruhlik, K.; Brown, J.R. (ed.), The Natural Philosophy of Leibniz, Springer, Dordrecht, 1985, pp. 276-277. En mi opinión, Arthur atina al caracterizar el apetito como este principio de cambio, pero no toma en cuenta las complicaciones que implica esta consideración para el resto del sistema leibniziano. 
Esto nos pone en una situación complicada: o bien se entiende que lo que se difunde en el tiempo son verdaderas unidades que constituyen a la mónada (y en este caso lo que se pierde es la individualidad de la mónada y del yo), o bien se entiende que la difusión no es más que una modificación de una realidad unitaria - la mónada-, en cuyo caso se tendría que concluir - por la analogía espacio-tiempo- que las mónadas no son sino modos del universo como totalidad.

La primera alternativa es inviable para Leibniz porque el concepto de mónada es estructurado a priori por la necesidad metafísica de que los fundamentos de la realidad sean unidades reales ${ }^{35} \mathrm{y}$, a posteriori, a partir de la intuición directa de la indivisibilidad del $y o^{36}$. Perder la unidad de la mónada implicaría echar por tierra el sistema monadológico en su totalidad. La segunda alternativa tampoco parece ser una opción para Leibniz, pues hacer de las mónadas, o de su posición, simples modificaciones del universo o del espacio absoluto implicaría una reducción de su pensamiento a un espinosismo inaceptable para él ${ }^{37}$.

Hay, así, dos elementos que ponen en jaque la analogía espacio-tiempo en el pensamiento leibniziano. El primero es la consideración en la Monadología de un principio de cambio interno en la mónada que supondría un tiempo que enmarcaría, absolutamente, los estados sucesivos de las mónadas. Esto no sólo introduciría a las sustancias en un tiempo absoluto, sino que volvería superfluas las definiciones del tiempo como el «orden de los fenómenos» o el «orden de las posibilidades sucesivas». El segundo es que los estados monádicos que se difunden en la duración no son ellos mismos individuos, sino que son las modificaciones de un individuo, lo cual discrepa con el tipo de difusión que representa el espacio.

\section{Algunos intentos de SOlución}

Tal vez sea ahora un buen momento para atender a lo que han dicho algunos scholars sobre estas complicaciones.

Como podría suponerse, la opinión de los comentaristas sobre el tema del tiempo está estrechamente ligada con la valoración que hagan del tema

35 «Lo que no es verdaderamente $u n$ ser no es, ciertamente, un ser». Leibniz a Arnauld, 30 de abril de 1687, A II, 2, p. 186; también cfr. Principes de la Nature et de la Grace, fondés en raison, §1-2, G VI, p. 598; Leibniz a Des Bosses, 14 de febrero de 1706, G II, p. 300; 11 de marzo de 1706, G II, p. 304.

36 «[P] odemos pensar en eso que se llama yo y considerar que esto o aquello está en nosotros; y así resulta que al pensar en nosotros, pensamos en el ser, en la sustancia, en lo simple y en lo compuesto...». Monadologie, §30, G VI, p. 612. "Es verdad que nosotros conocemos nuestra existencia por una intuición inmediata». Quelques remarques sur le livre de Mons. Lock intitulé Essay of Understanding, A VI, 6, p. 8. Cfr. También, Leibniz a De Volder, 30 de junio de 1704, GP II, p. 270; Leibniz a Lady Masham, mayo de 1704, G III, pp. 339-340.

37 Cfr. Leibniz a De Volder, 10 de noviembre de 1710, pp. 257-258. 
del espacio. En este sentido, aquéllos autores cuya lectura sobre la noción de espacio leibniziana no coincida con la descrita en la segunda sección de este trabajo, no coincidirán tampoco - por las mismas razones- con lo dicho aquí sobre el tiempo. Las discrepancias principales entre los comentadores se encuentran generalmente en el problema de ubicar al espacio y al tiempo, a la extensión y la duración, dentro de los tres niveles metafísicos del sistema leibniziano -monádico o real, fenoménico o semimental e ideal o mental-. No es el lugar aquí para discutir las diferentes interpretaciones que hay sobre el espacio en la filosofía leibniziana. Quisiera, en cambio, concentrarme en las discrepancias que hay sobre la interpretación de la analogía espacio-tiempo. En este sentido, no atenderé a los comentadores que difieren de mi posición en torno al tema del tiempo, por concebir al espacio de un modo diverso al mío, sino a aquellos que difieren en el modo de entender la relación entre el tiempo y el espacio en términos conceptuales dentro de la obra de Leibniz ${ }^{38}$.

Se ha hablado ya del problema que representan las afirmaciones de la Monadología en torno al cambio monádico. Para algunos, la solución a este dilema ha sido simplemente entender de un modo literal las afirmaciones leibnizianas y dar por hecho que la mónada es un sujeto de cambio y que, por tanto, la relación de ella con el tiempo es muy distinta a la de ésta con el espacio.

Rescher, por ejemplo, postula un «tiempo privado» y un «tiempo público». El tiempo privado es el que se deriva del cambio monádico presentado por Leibniz en la Monadología y representa el paso de un estado monádico a otro; el tiempo público es la coordinación armónica entre las mónadas por la cual es posible que los estados en una mónada se correspondan, en un instante determinado, con los estados de otra mónada ${ }^{39}$. Benson Mates parece tener una perspectiva semejante en la medida en que acepta un tiempo monádico y un tiempo derivado ${ }^{40}$. Anapolitanos, uno de los pocos que problematiza y analiza frontalmente el problema de la analogía entre espacio y tiempo, afirma que

38 Existe una discusión sobre la naturaleza del tiempo bastante desarrollada en la bibliografía secundaria, enfocada principalmente en la reductibilidad (o no) de las relaciones temporales a relaciones de causalidad. En esta dirección se pueden citar varios trabajos: ARthur, Richard, «Leibniz's Theory of Time», en: OKruhlik, K.; Brown, J.R. (eds.), The Natural Philosophy of Leibniz, Springer, Dordrecht, 1985, pp. 263-313; Cover, JAN, «Non-Basic Time and Reductive Strategies: Leibniz's Theory of Time», en: Studies in History of Philosophy of Science, vol. 28, núm. 2, 1997, pp. 289-318; Futch, M., Leibniz's Metaphysics of Time and Space, passim; Di Bella, Stefano, «Time, Contradiction and Change in Leibniz: Some Reflections», pp. 263-267; SiLva, CAmilo, "Y a-t-il vraiment une théorie causale du temps chez Leibniz?», en: LI, Wenchao, et al (eds.), «Für unser Glück oder das Glück anderer»Vorträge des X. Internationalen Leibniz-Kongress, vol. IV, Olms, Hildescheim, 2016, pp. 323-334. Sin embargo, el enfoque del presente trabajo no es el de dar luz sobre la relación entre causalidad y el tiempo, sino entre el tiempo, el espacio y las mónadas.

39 Cfr. Rescher, N., The Philosophy of Leibniz, Prentice Hall, New Jersey, 1967, p. 89.

40 Cfr. Mates, B., The Philosophy of Leibniz, Oxford Univ. Press, New York, 1986, pp. 228-229. Hay que tomar en consideración que Mates no descarta la analogía espacio-tiempo porque asume igualmente un espacio monádico y un tiempo monádico. 
Leibniz se equivoca en su valoración del tiempo como analogado del espacio y termina postulando un tiempo monádico real, habiendo negado antes el espacio monádico ${ }^{41}$. La posición de Lois Frankel es semejante a la de Anapolitanos en este sentido ${ }^{42}$.

Esta posición —la de la asimetría radical entre la relación mónada-espacio y la relación mónada-tiempo- tiene ciertas complicaciones. Es verdad que permite dar sentido y validez a las expresiones de la Monadología, pero no explica la ingente cantidad de textos en los que Leibniz habla del tiempo y el espacio como entidades derivadas, fenoménicas o ideales. Si hay un tiempo monádico, ¿cómo habría de diferir éste de la duración fenoménica? ¿en qué sentido ésta sería derivada respecto a aquélla? Y más aún: ¿qué significado tiene el hecho de que esta relación de derivación sea equivalente a la que hay entre las mónadas no espaciales y el extenso fenoménico?

Otra vía de solución es la adoptada por Michael Futch, quien sigue en buena medida la lectura de Robert Adams. Futch critica a aquéllos que colocan a la mónada directamente en el espacio o en el tiempo ${ }^{43}$, pero propone que un estado monádico tiene una posición en el tiempo exclusivamente por la posición que tiene dentro de la serie ordenada de los estados en la mónada. Esta afirmación es derivada de la tesis de Futch y Adams sobre el espacio y las mónadas, a saber, que las mónadas tienen una ubicación en el espacio que es derivada y está dada en función del cuerpo orgánico que poseen. Esta lectura asume que, si bien las mónadas no están en el espacio y en el tiempo, sí son «ubicables» dentro de los fenómenos espacio-temporales a través de su cuerpo orgánico. Si usáramos los términos de Rescher, se podría decir que Futch afirma que la posición de las mónadas sólo es asignable en el «tiempo público», esto es, en el devenir conjunto y armónico de las mónadas:

«Un estado monádico tiene una posición en el tiempo, y es ordenado temporalmente con otros estados monádicos sólo en virtud de la proyección en el mundo fenoménico espacio temporal, un mundo en el que los agrados fenoménicos están relacionados temporalmente entre ellos» ${ }^{44}$.

Soy de la opinión de que esta interpretación es incompatible con los textos leibnizianos, tanto por la parte del espacio como por la parte del tiempo. Las mónadas notienenubicación en el espacioyen el tiempo másque metafóricamente. El engaño en el que incurren estas interpretaciones es, precisamente, el mismo en el que incurre Rescher: el suponer que existe un «tiempo público». Aunque Futch niega que exista algo así como un «tiempo privado», piensa a la duración como un fenómeno único y compartido por las mónadas. La simple idea de un

41 Cfr. Anapolitanos, D., Leibniz: Representation, Continuity..., o.c., pp. 142-143. Esto no impide que su modelo simbólico sea también aplicado al tiempo.

42 Cfr. Frankel, Lois, "Leibniz on the Foundation of Space and Time», en: Nature and System, vol. 3, 1981, p. 93.

43 Cfr. Futch, Michael, Leibniz's Metaphysics of Time and Space, Springer, Berlín, 2008, pp. 162-163.

${ }^{44}$ Ibíd., pp. 167-170. 
«mundo fenoménico» en el que los estados monádicos «son proyectados» refleja ya una malinterpretación de la palabra fenómeno. No hay un «mundo de los fenómenos» precisamente porque éstos constituyen los estados internos de las mónadas. Si la duración es el orden de los fenómenos sucesivos, entonces la duración es el orden de los estados internos de la mónada. No hay publicidad alguna de esta ordenación. Es precisamente en este sentido en el que Leibniz puede decir que las mónadas no tienen ventanas y es también precisamente por esto que la Armonía Preestablecida es un requerimiento del sistema ${ }^{45}$ :

«Las mónadas no tienen en absoluto ventanas por las que pueda salir o entrar algo. Los accidentes no pueden desprenderse ni andar fuera de las sustancias, como hacían en otro tiempo las especies sensibles de los escolásticos. Así pues, ni sustancia ni accidente alguno puede entrar desde fuera en una mónada» ${ }^{46}$.

Considerar al tiempo o a la duración como algo «público» o como una cualidad de «el mundo de los fenómenos» es asignar a un accidente dos sustratos distintos. Esto rompería con el principio básico de la Monadología y vuelve estéril el esfuerzo de Adams y Futch por recomponer, a partir de ésta, la teoría sobre el tiempo.

\section{UNA SOLUCIÓN ALTERNATIVA}

Así las cosas, parece que hay dos temas que deben ser abordados para perfilar correctamente el tema del tiempo: por una parte, hay que reconciliar los textos en donde se equiparan tiempo y espacio con los fragmentos de la Monadología en donde se postularía un presunto cambio en la mónada. Como se ha visto, buena parte de los comentadores toman al pie de la letra las afirmaciones leibnizianas sobre el cambio monádico sin atender al problema que esto implica. Por otra parte, habría que atender una incongruencia que, hasta donde sé, no ha sido notada en la literatura crítica sobre el tema: el problema de que la difusión del tiempo no es una difusión de individuos, sino de diversos estados de un mismo individuo. Esta situación nos pone en una paradoja en la que, o bien se pierde la individualidad de la mónada y se cae en el laberinto del continuo, o bien se afirma a la mónada como una modificación del universo y se cae en el espinosismo.

45 McRae señala que hay una evolución en el pensamiento leibniziano en este sentido. Si bien en textos más tempranos, como la correspondencia con Arnauld (Cfr. Leibniz a Arnauld, septiembre de 1687, G II, p. 115), la Armonía Preestablecida parece considerarse como la condición de posibilidad del espacio y el tiempo, en la etapa monadológica estos parecen ser tratados como condiciones de los fenómenos internos de las mónadas (Cfr. Leibniz a Clarke, 18 de agosto de 1716, G VII, p. 400). Cfr. McRae, Robert: «Time and the Monad», en: Woolhouse, Roger (ed.), Gottfried Wilhelm Leibniz. Critical Assessments. Vol. III., Routledge, London, 1994, p. 107.

46 Monadología, §7, G VI, pp. 607-608. 
Respecto al primer problema, es de utilidad poner en contexto las afirmaciones de la Monadología. Si se toman literalmente esas expresiones, se vuelve en efecto implausible que las mónadas estén fuera del tiempo. Opino, en consecuencia, que no se deben de considerar literalmente estas afirmaciones.

La Monadología es uno de los textos más sintéticos de Leibniz; en escasos 90 parágrafos el sabio alemán enuncia prácticamente todas sus tesis más relevantes de Metafísica, Teodicea, Epistemología y Filosofía de la Naturaleza. Este texto fue pensado por Leibniz, probablemente, como un trabajo divulgativo que, a través de Nicolas Remond (a quien estaba dirigido), pretendía darse a conocer en los círculos aristocráticos de París. Con estos antecedentes, es normal que Leibniz no pudiera, ni le conviniera, adelantar sus tesis más radicales y polémicas sobre la relación entre las sustancias, el espacio y el tiempo.

Ahora bien, esta explicación contextual no nos exime de reconciliar las tesis que hemos descrito sobre el tiempo y la consideración de un principio de cambio en la mónada. Leibniz podrá estar siendo divulgativo en la Monadología, pero dudo que esté siendo impreciso ${ }^{47}$. Descartar arbitrariamente como falsos un conjunto de textos por parecer incompatibles a otros no es deshacer el nudo gordiano: es cortarlo ciegamente.

En muchos textos, principalmente aquellos relacionados con la dinámica, Leibniz propone que la esencia de la mónada es la Fuerza. La Fuerza puede ser primitiva o derivativa, activa o pasiva. Me concentraré ahora en las fuerzas activas, tanto primitivas como derivativas (dejando a un lado sus contrapartes pasivas, porque las activas son precisamente las que están relacionadas con el cambio del que habla Leibniz en la Monadología).

Con su a veces desenfadado eclecticismo terminológico, Leibniz no duda en llamar entelecheia hê prôte a la Fuerza primitiva activa, que «responde al alma o forma sustancial, pero por ello mismo no atañe sino a las causas generales, que no pueden ser suficientes para explicar los fenómenos» ${ }^{48}$. En este caso, la selección del término entelequia, un término que denota actualidad y completud, no es en lo absoluto arbitraria. Leibniz busca, precisamente, que el principio de cambio de la mónada no sea él mismo modificado ${ }^{49}$. Cualquier

47 Contra la célebre hipótesis de Bertrand Russell de los «diferentes Leibniz». Russell afirma que el sabio alemán, como diplomático que era, moldeaba su sistema al gusto de los Príncipes y nobles a quien se dirigía para no salir de la ortodoxia de la época; esto generará que el sistema leibniziano adolezca de serias inconsistencias cuando se atiende a textos públicos y privados. Cfr. Russell, Exposición crítica..., pp. 19-21.

48 Specimen Dynamicum I, GM VI, p. 236.

49 Es interesante que ya Aristóteles caracterizaba al alma como un motor inmóvil (cfr. De anima, I, cap. 3, 406a-407b) y afirmaba que ésta se mueve y posee un lugar sólo accidentalmente (cfr. De anima, I, cap. 4, 408a, 30). Para Leibniz la mónada no posee posición en el espacio sino metafóricamente. «Estimo útil para una investigación fundamental de las cosas la explicación de todos los fenómenos por las solas percepciones de las Mónadas concordantes entre sí, dejando aparte la substancia corpórea. Y con esta manera de explicar, el espacio deviene el orden de los fenómenos coexistente, como el tiempo es el orden de los fenómenos sucesivos; y no hay ninguna proximidad ni distancia espacial o absoluto entre las Mónadas, 
modificación tendría que estar ya incluida en esta entelequia: todos los estados de la mónada están ya en ella, no desde siempre, sino desde fuera del tiempo.

Si las fuerzas primitivas no explican directamente los fenómenos, las fuerzas derivativas sí se encuentran precisamente en este nivel. La fuerza derivativa es el objeto propio de la dinámica, y es la causa directa del movimiento en los fenómenos, cuya medida establecerá Leibniz con la famosa fórmula de $m v^{2}$. Esta fuerza fenoménica ya es de poco interés para nosotros, pues no puede sino ser fruto de alguna combinación conceptual de los términos de extensión y duración fenoménicos. Me interesa, más bien, la relación entre la fuerza primitiva y la fuerza derivativa.

Leibniz es ambiguo en este tema y el solo estudio de esta relación merecería un tratamiento aparte ${ }^{50}$. Baste con referir la idea leibniziana de que la fuerza derivativa es una modificación de la fuerza primitiva. Esto es: la entelequia es una forma, una ley que se ve instanciada y delimitada. La ley contiene todos los estados posibles de la mónada, pero éstos no se ven desplegados sino en la modificación, y el resultado de dicha modificación es la fuerza derivativa; en los fenómenos, esa ley se ve instanciada por el movimiento ${ }^{51}$. Del mismo modo en que una ecuación establece una ley —una función-cuya naturaleza y subsistencia es independiente de la verificación gráfica de sus instancias, así la entelequia precede al despliegue espacio-temporal de sus estados representativos.

Revisemos, pues, lo que Leibniz dice en la Monadología, a la luz de estas acotaciones:

10. Doy también por sentado que todo ser creado está sujeto a cambio y, en consecuencia, también la mónada creada, e incluso que dicho cambio es continuo en cada una.

11. De lo que acabamos de decir se sigue que los cambios naturales de las mónadas proceden de un principio interno, puesto que una causa externa no puede influir en su interior (Teodicea, §§ 396 y 400).

12. Pero también es preciso que, además del principio del cambio, haya un detalle de lo que cambia [detail de ce qui change], que efectúe, por así decir, la especificación y la variedad de las sustancias simples.

13. Ese detalle debe envolver una multitud en la unidad o en lo simple. Pues como todo cambio natural se realiza por grados, algo cambia y algo permanece; y por consiguiente en la sustancia simple tiene que haber una pluralidad de afecciones y de relaciones, aunque carezca de partes ${ }^{52}$.

y decir que están conglomeradas en un punto o diseminadas en el espacio es usar algunas ficciones de nuestro espíritu porque nos sería grato imaginar aquello que solamente puede ser concebido». Leibniz a Des Bosses, 16 de junio de 1712, G II, p. 451.

50 Un esbozo de este tratamiento lo he intentado hacer en RuIz-Gómez, LEONARdo, «Fuerza primitiva y fuerza derivativa en G.W. Leibniz» en: Tópicos, vol. 48, 2015, pp. 141-168.

51 Hay que notar que para Leibniz todo cambio es reductible en los fenómenos a movimientos locales; con esta idea se mantiene fiel al paradigma mecanicista, cuya filiación Leibniz confiesa en reiteradas ocasiones. Cfr. Considérations sur les Principes de Vie..., G VI, p. 541; Principium ratiotinandi fundamentale, C, p. 12.

52 Monadologie, §10-12, G VI, p. 608. 
Ciertamente Leibniz habla de que la mónada está sujeta a cambio, pero distingue entre el principio de cambio y el «detalle de aquello que cambia». Agrega después que este detalle es lo que permite la especificación y la variedad de sustancias. Pero habíamos visto antes que es el situs monádico —el punto de vista de las mónadas - lo que permite su individuación en términos representativos. Esto indicaría que este «detalle» debe estar relacionado con el punto de vista de la mónada; esto es, con el fundamento, según lo visto, de la extensión y la duración fenoménica. Ciertamente las percepciones en la mónada van cambiando (porque va cambiando el situs), pero la esencia de la mónada, esto es, la entelequia o principio del cambio, no cambia ella misma ${ }^{53}$. Así como $y=x^{2}$ no cambia cuando se recorren gráficamente los puntos de una parábola (sus instancias), así la mónada o fuerza primitiva no cambia cuando se modifica en sus percepciones.

Esta consideración del cambio en términos derivativos soluciona el primero de los problemas que apuntábamos antes: el cambio tampoco pertenece a la entelequia monádica; al igual que el tiempo, pertenece a los fenómenos. Que en la mónada haya un principio de cambio no pone en riesgo la anterioridad ontológica de la mónada respecto al tiempo. Es más, revela la cualidad que tiene la mónada de fundamento respecto a las entidades derivadas del espacio, el tiempo y el movimiento.

Esta misma consideración podría ser de ayuda para dar razón de la segunda aporía. Se había explicado que en la difusión que engendraba la extensión no se podía asumir a las mónadas como modos, ya que eso implicaría caer en el espinosismo. Asumir, en cambio, a los estados de las mónadas como individuos pulverizaría la noción de mónada. Me parece que entender a la fuerza derivativa como modificación de la entelequia ayuda a salir de esta disyuntiva: los diversos estados de las mónadas son modificaciones de la entelequia, no son mónadas en sí mismas. Esto aplica no sólo a la duración, como se ha explicado, sino también a la extensión. La mónada no es una modificación; la modificación es el punto de vista desde el que percibe el universo y el estado actual desde el que lo percibe el resto de las mónadas. Este punto de vista es una modificación de la mónada que percibe, pero no hace del resto de las mónadas modos del espacio absoluto. La difusión que genera la extensión y la duración es la modificación de una sola mónada, no hace de la pluralidad de las mónadas distintas modificaciones de un ser omniabarcante.

53 Richard Arthur relaciona con mucho acierto esta idea leibniziana con una afirmación de un texto muy anterior: el Pacidius Philalethi, de 1676. Ahí Leibniz intenta solucionar el problema del movimiento continuo apelando a una causa inmóvil del movimiento: «Por lo tanto, aquello por lo que los cuerpos son movidos y trasladados, no es el cuerpo mismo, sino una causa superior que, al actual, permanece inmutable, a saber, Dios». Pacidius Philalethi, A VI, 3, p. 567 (Mi traducción). Para Arthur, en el Leibniz maduro esta causa inmóvil del movimiento pasará de ser Dios a ser la mónada. Cfr. ARTHUR, R., «Leibniz's Theory of Time», en: Okruhlik, K.; Brown, J.R. (ed.), The Natural Philosophy of Leibniz, o.c., p. 297. 


\section{Algunas anotaciones finales}

Convendría en este punto establecer algunas consecuencias que tiene la perspectiva que he defendido en este trabajo.

Hay, ciertamente, una interesante disparidad entre el espacio y el tiempo que debe ser analizada; por ejemplo, al estar definido por la «coexistencia», el espacio parece ser un concepto lógicamente posterior al del tiempo ${ }^{54}$. Pero esta jerarquización no saca al tiempo del ámbito fenoménico e ideal. La anterioridad del tiempo sobre el espacio está en el orden fenoménico y no nos obliga a postular un tiempo monádico fundamental.

Espero haber mostrado así que la teoría leibniziana del tiempo adquiere una riqueza particular cuando se observa en paralelo — siguiendo la sugerencia del mismo Leibniz - con la teoría del espacio. Sin embargo, es de suma importancia establecer los límites de este paralelismo, pues en ellos se encuentran matices que resultan incluso más valiosos para la comprensión de estas nociones que la analogía que los identifica. Los problemas que han surgido al intentar esclarecer esta relación nos han puesto de relieve la importancia de ciertos elementos de la dinámica leibniziana para comprender el corazón mismo de la teoría monadológica. Esto apunta también a una congruencia doctrinal que denota el carácter sistemático del pensamiento del bibliotecario de Hannover: un síntoma de que el sabio alemán no sólo abordó una variedad ingente de frentes, sino que lo hizo con un espíritu de coherencia y articulación holística admirables.

BiBLIOGRAFÍA

\section{Obras de Leibniz.}

A= Sämtliche Schriften und Briefe, Darmstadt-Berlin: Berlin Academy 1923.

$\mathrm{G}=$ Die philosophischen Schriften von Gottfried Wilhelm Leibniz, ed. C. I. Gerhardt, Berlin: Weidman 1875-90.

GM= Leibnizens matematische Schriften, ed. C. I. Gerhardt, Berlin: Halle 1849-1863.

$\mathrm{C}=$ Opuscules et fragments inédits de Leibniz, ed. Louis Couturat, Paris: Félix Alcan 1903.

Las traducciones empleadas, si no se señala lo contrario, fueron tomadas de:

G.W. Leibniz: Obras filosóficas y científicas, Granada: Comares 2007 (a la fecha).

\section{Literatura Secundaria}

Anapolitanos, Dionysios, Leibniz: Representation, Continuity and the Spatiotemporal, Kluwer Academic, Dordrecht, 1999.

54 Contra lo dicho en Rescher, N., Leibniz's Metaphysics of Nature, Western Ontario Univ. Press, Dordrecht, 1981, pp. 96-97. 
ARIstóteles, Acerca del alma, Gredos, Madrid, 2003.

ARTHur, Richard, «Leibniz's Theory of Time», en: Okruhlik, K.; Brown, J.R. (ed.), The Natural Philosophy of Leibniz, Springer, Dordrecht, 1985, pp. 263-313.

Cover, JAN, «Non-Basic Time and Reductive Strategies: Leibniz's Theory of Time», en: Studies in History of Philosophy of Science, vol. 28, núm. 2, 1997, pp. 289-318

Своскет, Тімотну, "Space and Time in Leibniz's Early Metaphysics», en: The Leibniz Review, vol. 18, 2008, pp. 41-79.

Di Bella, Stefano, «Time, Contradiction and Change in Leibniz: Some Reflections», en: Li, Wenchao, et al (eds.), "Für unser Glück oder das Glück anderer»Vorträge des X. Internationalen Leibniz-Kongress, vol. IV, Olms, Hildescheim, 2016, pp. 263-267

Frankel, LoIs, «Leibniz on the Foundation of Space and Time», en: Nature and System, vol. 3, 1981, pp. 127-147.

Futch, Michael., Leibniz's Metaphysics of Time and Space, Springer, Dordrecht, 2008.

GARBER, DANIEL, «Dead Force, Infinitesimals and the Mathematicization of Nature», en: Goldenbaum, Ursula; Jesseph, Douglas (eds.), Infinitesimal Differences. Controversies between Leibniz and his Contemporaries, De Gruyer, Berlin, 2008, pp. 281-306.

Leibniz: Body, substance, Monad, Oxford Univ. Press, Oxford, 2009.

Hartz, Glenn; Cover, J., "Space and Time in the Leibnizian Metaphysics», en: Woolhouse, Roger (ed.), Gottfried Wilhelm Leibniz. Critical Assessments. Vol. III, Routledge, London, 1994, pp. 295-316.

Mates, Benson, The Philosophy of Leibniz, Oxford Univ. Press, New York, 1986.

McGuire, J. E., "Labrynthus Continuii': Leibniz on Substance, Activity and Matter», en Turnbull, Robert; Machamer, Peter (eds.), Motion and Times, Space and Matter, Ohio State Univ. Press, Columbus, 1976, pp. 290-326.

McRae, Robert: «Time and the Monad», en: Woolhouse, Roger (ed.), Gottfried Wilhelm Leibniz. Critical Assessments. Vol. III., Routledge, London, 1994, pp. 104-111.

Rescher, Nicolas, The Philosophy of Leibniz, Prentice Hall, New Jersey, 1967.

Leibniz's Metaphysics of Nature, Western Ontario Univ. Press, Dordrecht, 1981.

Ruiz-Gómez, LeonARdo, El concepto leibniziano de espacio. La polémica con Clarke y el newtonianismo, EUNSA, Pamplona, 2014.

«Fuerza primitiva y fuerza derivativa en G.W. Leibniz», en: Tópicos, vol. 48, 2015, pp. 141-168.

Russell, Bertrand, Exposición crítica de la Filosofía de Leibniz, Buenos Aires, Siglo XX, 1977.

SiLva, Camilo, «Y a-t-il vraiment une théorie causale du temps chez Leibniz?», en: Li, Wenchao, et al (eds.), «Für unser Glück oder das Glück anderer» Vorträge des X. Internationalen Leibniz-Kongress, vol. IV, Olms, Hildescheim, 2016, pp. 323-334.

Winterbourne, A.T., "On the Metaphysics of Leibnizian Space and Time», en: Woolhouse, Roger (ed.), Gottfried Wilhelm Leibniz. Critical Assessments. Vol. III, Routledge, London, 1994, pp. 62-75.

Universidad Panamericana. Campus México.

Facultad de Filosofía

LEONARDO RUIZ-GómEZ

leruiz@up.edu.mx

[Artículo aprobado para publicación en diciembre de 2016] 